\title{
Enquête de consommation alimentaire en zone CEMAC : Cas de la république du Congo et du Gabon
}

\author{
Hugues R. PAMBA BOUNDENA ${ }^{1}, 2^{\star}$, Raphaël BIKANGA ${ }^{2}$, Vital MANANGA ${ }^{1}$, Thomas SILOU1. \\ 'Laboratoire du Pôle d'Excellence Régional (AUF) en "Alimentation et Nutrition", Équipe Pluridisciplinaire de \\ Recherche en Alimentation et Nutrition (E.P.R.A.N.), BP : 389 Brazzaville - Congo Email : eprancongo@yahoo.fr \\ 2Laboratoire de Chimie des Substances Naturelles et Synthèses Organométalliques (LASNSOM), Faculté des \\ Sciences, Université des Sciences et Techniques de Masuku (USTM), BP : 941, Franceville - Gabon \\ ${ }^{*}$ Correspondant : hugues_pamba@yahoo.com
}

Original submitted in on $4^{\text {th }}$ May 2016. Published online at www.m.elewa.org on 31 $1^{\text {st }}$ August 2016 http://dx.doi.org/10.4314/jab.v104i1.3

\section{RESUME}

Objectif : L'objet de cette étude est de comparer, à partir de deux enquêtes nationales (au Congo et au Gabon), les pourcentages de la population concernée par la consommation d'huiles alimentaires.

Méthodologie et Résultats : Cette étude analyse les éléments suivants : (i) le niveau sociodémographique à travers l'âge, le sexe, le statut matrimonial et le statut professionnel. (ii) le niveau de la consommation des oléagineux à l'état brut et semi brut et/ou transformés en huile à travers les modalités d'approvisionnement, de conservation et de consommation. (iii) la dynamique de la consommation réelle des différents produits alimentaires, (iv) le niveau de dépendance alimentaire de la sous région et (v) les préférences alimentaires des populations. Les analyses effectuées à partir des enquêtes sur les ménages ont été désagrégées par milieu de résidence et par niveau de revenu, afin de permettre un meilleur ciblage dans la conception des filets de sécurité alimentaire. Premièrement, au niveau de la transformation, les résultats de l'enquête montrent qu'au Congo $87,8 \%$ des sujets enquêtés ne transforment pas les oléagineux et $5,8 \%$ transforment les noix de palme contre $64,7 \%$ des sujets enquêtés qui transforment les oléagineux et notamment les noix de palme au Gabon. Deuxièmement, au niveau de la consommation, $15,1 \%$ des sujets enquêtés au Congo consomment des amandes et/ou pâtes de péké, 95,7\% consomment des noix de palme (ou mwambe), 96,4\% consomment des graines et/ou pâtes d'arachide, 87,8 \% consomment des graines et/ou pâtes de courge, 89,2 \% consomment du safou et enfin 2,9\% consomment les autres oléagineux tels que l'avocat ou les noisettes. Par contre, au Gabon, 93,6\% des sujets enquêtés consomment les amandes et/ou pâtes d'odika, $94 \%$ consomment les noix de palme, $88,5 \%$ consomment les graines et/ou pâtes d'arachide, $78,9 \%$ consomment les graines et/ou pâtes de courge, $95,9 \%$ consomment les atangas et enfin 56,9\% consomment les autres oléagineux tels que les noisettes et l'avocat.

Conclusion et application des résultats: Les analyses effectuées à partir des deux enquêtes a permis d'observer que beaucoup de ménages enquêtés consomment les mêmes types d'oléagineux dont certaines appellations peuvent différer. Ainsi, la valorisation de ces produits locaux peut contribuer au développement de ces deux pays. Cette étude a été menée afin de guider les décideurs dans la conception d'une réglementation adéquate de sécurité alimentaire.

Mots-Clés : Enquête de consommation, oléagineux, Congo, Gabon 


\title{
Boundena et al . J. Appl. Biosci. 2016 Enquête de consommation alimentaire en zone CEMAC : Cas de la
} république du Congo et du Gabon

Household food consumption in CEMAC zone: Cases of Congo republic and Gabon

\begin{abstract}
Objective: The objective of this study is to compare, from two national surveys (in Congo and Gabon), percentages of the population concerned with the consumption of oilseeds.

Methodology and Results: This study analyzed the following: (i) the socio-demographic level across age, sex, marital status and employment status. (ii) the level of consumption of oilseeds in the rough and semi raw and / or processed oil through the supply arrangements, conservation and consumption. (iii) the dynamics of the actual consumption of different food products, (iv) the level of food dependency in the region and (v) food preferences of the population. The analyses carried out from household surveys were disaggregated by place of residence and income level, to enable better targeting in designing food safety nets. First, at the processing level, the results of the survey show that in Congo $87.8 \%$ of the respondents do not transform oilseeds and $5.8 \%$ transform palm nuts against $64.7 \%$ of the respondents who transform oilseeds including palm nuts in Gabon. Second, the level of consumption, $15.1 \%$ of subjects surveyed in Congo consume almonds and / or Peke pasta, $95.7 \%$ consume palm nuts (or Mwambe), $96.4 \%$ use the seeds and / or peanut paste, $87.8 \%$ consume the seeds and / or squash pasta, $89.2 \%$ use safou and finally $2.9 \%$ use other oilseeds such as avocado or nuts. By cons, Gabon, $93.6 \%$ of the respondents consume almonds and / or pastes odika, $94 \%$ consume palm nuts, $88.5 \%$ consume the seeds and / or peanut paste, $78.9 \%$ consume the seeds and / or squash pasta, $95.9 \%$ consume atangas and finally $56.9 \%$ use other oilseeds such as nuts and avocado.

Conclusion and implementation of the results : Analyses observed that many surveyed households consume the same types of oilseeds, some names may differ. Thus, the valuation of these local products can contribute to the development of both countries. This study was conducted to guide policy makers in designing adequate regulation of food safety.
\end{abstract}

Keywords : Investigation of consumption, oilseeds, Congo, Gabon.

\section{INTRODUCTION}

L'objet de cette étude est de comparer, à partir de deux enquêtes nationales de consommation alimentaire au Congo et au Gabon, les pourcentages de transformation et de consommation à l'état brut et/ou semi brut des oléagineux par les populations de ces deux pays. Les oléagineux concernent plusieurs groupes d'aliments et sont fonction des préférences des populations de la sous-région. La filière des oléagineux tels que l'odika, les noix de palme, les graines d'arachide, les atangas ou le safou, a traversé une période de turbulence, caractérisée par de larges fluctuations de prix, qui ont tendu vers le haut par rapport aux années 20062007. Après la flambée puis la chute spectaculaire des prix en 2008, par exemple le tas des noix de palme à $200 \mathrm{~F}$ était donné à $100 \mathrm{~F}$, les prix se sont engagés sur une trajectoire résolument à la hausse en 2009, reflétant ainsi le resserrement progressif des approvisionnements mondiaux, la reprise de l'accroissement de la demande (à l'issue de la crise économique mondiale) et de solides intentions d'achats de la part des principaux pays importateurs (FAO, 2011). Malgré une méthodologie très identique, notamment en ce qui concerne les questionnaires, les groupes d'aliments ont été classés selon la même hiérarchie dans les deux enquêtes La production d'huile de palme se répartit entre un secteur industriel avec des plantations satellites des agro-industries ou des plantations villageoises encadrées et un secteur villageois (Montfort., 2005). Les tensions entre l'offre et la demande provoquent souvent une augmentation généralisée des prix, réduisant le pouvoir d'achat des populations démunies, rurales ou urbaines, et les mettant en situation d'insécurité alimentaire chronique ou conjoncturelle selon les cas (FAO. 2004a). La consommation alimentaire des pays de la sous région Afrique-centrale est basée principalement sur l'arachide, la noix de palme, l'amande de péké (Congo) ou odika (Gabon) la graine de courge et le safou ou atanga avec une contribution calorifique de $63 \%$ en moyenne 

république du Congo et du Gabon

(DEGBIA D., 2010). Elle est plus diversifiée au Cameroun, en République Démographique du Congo, au Congo et au Gabon. Le régime alimentaire en termes de consommation en macronutriments (glucides, protéines et lipides) est déséquilibré pour l'ensemble des pays de la sousrégion. La transformation artisanale concerne les plantations villageoises éloignées des zones d'influence des agro-industries La production est destinée au marché local et les rendements d'extractions ont plus faibles que dans le secteur industriel (MONTFORT M-A, 2005). Au Tchad, le régime alimentaire est principalement basé sur les

\section{METHODOLOGIE DE L'ENQUETE}

Zone d'enquête : La zone d'étude a concerné toute la ville de Brazzaville pour le Congo et les principales villes de six (6) provinces du Gabon à savoir: Libreville (province de l'Estuaire), Franceville (Haut-Ogooué), Lambaréné (Moyen-Ogooué), Makokou (Ogooué-lvindo), Koula-Moutou (Ogooué-Lolo) et Oyem (Woleu-Ntem)

Caractéristique démographique de l'enquête : Cette enquête a pris en compte les caractéristiques sociodémographiques en relation avec la consommation des oléagineux locaux à l'état brut ou semi brut et les oléagineux locaux transformés en huiles par les ménages enquêtés. Au niveau sociodémographique les variables étudiées sont: l'âge, le statut matrimonial et le statut professionnel. Au niveau de la consommation des oléagineux à l'état brut ou semi développés et/ou transformés en huile, ce sont les modalités d'approvisionnement, de conservation et de consommation qui ont fait l'objet de l'étude.

Type d'enquête: II s'agit d'une enquête de type transversale, avec un sondage à deux (2) degrés pour le tirage au sort des zones de dénombrement, des blocs et des unités statistiques au niveau des parcelles retenues dans la zone de dénombrement.

Mode de collecte de données : Les données ont été collectées à travers des questionnaires pré-codés pour permettre une transcription sur le support informatique.

Base de sondage : La population source de l'enquête était constituée des ménages habitant les villes enquêtées. L'unité statistique est le ménage (ensemble de personnes apparentées ou non partageant habituellement les mêmes repas, reconnaissant l'autorité d'un même individu appelé chef de ménage, et habitant dans la même maison) Le choix du ménage comme unité statistique a permis de recueillir l'information désirée (information sur le ménage et sur les membres du céréales (sorgho et mil) et les racines de tubercules (manioc, igname), complété par des légumineuses. La consommation de produits animaux et de fruits et légumes, aliments riches en micronutriments, est faible. Au niveau national, les disponibilités alimentaires du pays couvrent à peine les besoins énergétiques moyens de la population, et en raison des disparités dans l'accès aux aliments plus d'un tiers de la population est sous-alimentée. En conséquence, de nombreux ménages doit faire face à une insécurité alimentaire récurrente et parfois très sévère (FAO, 2009).

ménage) en interrogeant uniquement la personne qui s'occupe habituellement des repas II n'existait pas de critères d'exclusion pour qu'un ménage soit éligible La base de sondage pour la réalisation du tirage est celle de données statistiques du recensement général de la population et de l'habitat (RGPH, 2010). Dans ce recensement, chaque arrondissement a été subdivisé en zone de dénombrement Le tirage prend en compte les listes des zones de dénombrement et des blocs disponibles. Dans chaque ville, en l'absence des données récentes sur les effectifs des populations, les seuls renseignements disponibles utilisables pour l'enquête ont été la carte de la situation géographique des zones de dénombrement, la population totale Le nombre des zones de dénombrement retenus a été proportionnel au poids démographique. Des secteurs ont été ensuite définis pour la ville enquêtée (zone enquêtée). La nature et le nombre de secteurs dépendent des données disponibles (carte de la ville, recensement), et des zones de dénombrement qui sont des zones géographiques avec la population correspondante Toutes les zones de dénombrement ont été identifiées et les zones de dénombrement de travail sélectionnées par tirage aléatoire (au sort) de la première zone d'enquête ou bloc ( $2^{e}$ degré) le plus proche du point de chute.

Taille de l'échantillon : La taille de l'échantillon a été de 140 ménages pour la ville de Brazzaville (Congo) et de 218 ménages pour les 6 provinces enquêtées au Gabon Déroulement de l'enquête: Au plan pratique, la capacité journalière a été fixée à 6 ménages pour tenir compte des déplacements et de la charge de travail. En cas de collecte insuffisante des données, la méthode de proximité géographique a été privilégiée, pour compléter ou reprendre le recueil d'informations Le premier travail a consisté à vérifier les cartes des sections contenant les 
zones de dénombrement, dans la mesure du possible, afin de connaître le lieu de travail. Au terme de cette mise au point et vérification, l'enquête s'est déroulée de sorte que l'enquêteur s'est chargé de travailler auprès de tous les ménages relevant de la zone géographique regroupant l'ensemble des zones de dénombrement. Pour chaque ménage recensé, l'enquêteur a eu pour tâche d'évaluer les caractéristiques du ménage, l'approvisionnement, la conservation ou le stockage des oléagineux locaux à l'état brut et semi brut et des oléagineux locaux transformés en huile ainsi que la consommation de ces derniers. Les questionnaires relatifs aux ménages consommateurs /non consommateurs ont été soumis aux personnes qui s'occupent habituellement des repas. Tous les autres problèmes rencontrés (accessibilité, refus) ont été notés. Une maison où la ménagère principale était absente, ou bien qui refusait de répondre à l'interview a été aussitôt remplacée par la maison la plus proche, par proximité géographique, dans le sens du pas de progression de l'enquête. La bonne ou employée de maison qui s'occupait des repas a été interrogée même en présence de la maîtresse de maison. Dans chaque parcelle, une seule maîtresse de maison a été interrogée Dans le cas d'un immeuble, chaque appartement est considéré comme une maison. Ces conditions ont été respectées afin d'assurer une représentativité cohérente. Les interviews ont été réalisées en langue "française". Le point de départ de chaque zone à enquêter a été l'intersection des rues la plus proche du point de chute défini .La première parcelle pour commencer l'interview a été déterminée par la méthode pile ou face (avec une pièce de monnaie) Aucun critère d'exclusion pour qu'un ménage ne soit éligible n'a été retenu Les maisons ont été enquêtées de proche en proche.

Informations collectées: L'identité des personnes enquêtées, et les modalités de consommation des oléagineux locaux à l'état brut et semi brut et les oléagineux locaux transformés en huile ont été appréciées au moyen d'une enquête alimentaire par questionnaire. Ainsi le questionnaire portait sur les modalités d'approvisionnement, de stockage ou de conservation et de consommation par la méthode des listes des questions pré-codés. Les performances techniques sur le terrain étaient d'un pas de cinq (5) maisons après la première parcelle enquêtée, une fois la zone d'étude sélectionnée, en tenant compte des ménages ayant des procédés technologiques traditionnels mis en œuvres par les producteurs d'huile dans des ateliers artisanaux. Au total, deux enquêtes ont été réalisées, ces enquêtes reposaient sur l'approvisionnement, la conservation ou le stockage des oléagineux locaux à l'état brut et semi brut et des oléagineux locaux transformés en huile, à savoir un questionnaire à choix non forcés et une proportion aux sujets des thèmes comportant chacun plusieurs explications. Toutefois une pré-enquête sur 20 ménages a été nécessaire pour ajuster certaines questions et valider le questionnaire (MONTFORT M-A, 2005).

Traitement et analyse des données : Le traitement des données récoltées ainsi que la saisie et la production des tableaux bruts ont été réalisés à l'aide des logiciels Excel 2007, EPI-INFO 604 d fr-2001.

\section{RESULTATS ET DISCUSSION \\ Pratique des ménages et consommation des oléagineux \\ A. Cas du Congo}

Tableau 1 : Existence et connaissances des ménages par rapport à la transformation des oléagineux

\begin{tabular}{l|c|c|c|c}
\hline & Caractéristiques & Effectif & $\%$ & Valeur de $\mathbf{X} 2$ et $\mathbf{p}$ \\
\hline Ménages transformant ou & Oui & 1 & 0,7 & So $=32,287$ \\
non les oléagineux & Non & 122 & 87,8 & $\mathrm{P}<0,001$ \\
& Autres & 16 & 11,5 & \\
\hline Type d'oléagineux & Noix de palme & 8 & 5,8 & So $=2,903$ \\
transformés & Nsp & 131 & 94,2 & $\mathrm{P}<0,001$ \\
\hline Connaissance des & Oui & 6 & 4,3 & So $=33,182$ \\
transformateurs & Non & 119 & 85,6 & $\mathrm{P}<0,001$ \\
environnants & Nsp & 14 & 10,1 & \\
\hline
\end{tabular}

Nsp : Non spécifié 
Boundena et al. J. Appl. Biosci. 2016 Enquête de consommation alimentaire en zone CEMAC : Cas de la république du Congo et du Gabon

Le Tableau 1 montre au total que $87,8 \%(p<0,001)$ des sujets enquêtés ne transforment pas les oléagineux alors $11,5 \%$ n'ont pas spécifié leurs réponses De même $5,8 \%$ seulement transforment les noix de palme Une grande majorité des sujets enquêtés $(85,6 \% ; p<0,001)$ ne se soucient pas d'avoir des connaissances sur les produits consommés

Tableau 2 : Principaux types d'oléagineux locaux consommés à l'état brut ou semi brut

\begin{tabular}{|c|c|c|c|c|}
\hline Type d'oléagineux consommés & Caractéristiques & Effectif & $\%$ & Valeur de $x^{2}$ et $p$ \\
\hline $\begin{array}{l}\text { Amandes et lou pâte de péké } \\
(\mathrm{N}=139)\end{array}$ & $\begin{array}{l}\text { Oui } \\
\text { Non } \\
\text { Nsp }\end{array}$ & $\begin{array}{c}21 \\
117 \\
1\end{array}$ & $\begin{array}{c}15,1 \\
84,2 \\
0,7\end{array}$ & $\begin{array}{l}\text { So }=55,190 \\
P<0,001\end{array}$ \\
\hline Noix de palme et/mwambe & $\begin{array}{l}\text { Oui } \\
\text { Non } \\
\text { Nsp }\end{array}$ & $\begin{array}{c}133 \\
3 \\
3\end{array}$ & $\begin{array}{c}95,7 \\
2,2 \\
2,2\end{array}$ & $\begin{array}{c}\text { So }=56,542 \\
P<0,001\end{array}$ \\
\hline Graines et/ou pâte d'arachide & $\begin{array}{l}\text { Oui } \\
\text { Non } \\
\text { Nsp }\end{array}$ & $\begin{array}{c}134 \\
1 \\
4\end{array}$ & $\begin{array}{c}96,4 \\
0,7 \\
2,9\end{array}$ & $\begin{array}{l}\text { So }=60,998 \\
P<0,001\end{array}$ \\
\hline Graines et/ou pâte de courge & $\begin{array}{l}\text { Oui } \\
\text { Non } \\
\text { Nsp }\end{array}$ & $\begin{array}{c}122 \\
14 \\
3\end{array}$ & $\begin{array}{c}87,8 \\
2,2 \\
10,1\end{array}$ & $\begin{array}{l}\text { So }=37,215 \\
P<0,001\end{array}$ \\
\hline Safou & $\begin{array}{l}\text { Oui } \\
\text { Non } \\
\text { Nsp }\end{array}$ & $\begin{array}{c}124 \\
12 \\
3\end{array}$ & $\begin{array}{c}89,2 \\
8,6 \\
2,2\end{array}$ & $\begin{array}{c}\text { So }=38,838 \\
P<0,001\end{array}$ \\
\hline Autres oléagineux & $\begin{array}{l}\text { Oui } \\
\text { Non } \\
\text { Nsp }\end{array}$ & $\begin{array}{c}4 \\
131 \\
4\end{array}$ & $\begin{array}{c}2,9 \\
94,2 \\
2,9\end{array}$ & $\begin{array}{c}\text { So }=60,682 \\
P<0,001\end{array}$ \\
\hline
\end{tabular}

Dans le tableau 2, on constate que $84,2 \%$ ( $n=117$; $p<0,001$ ) de sujets ne consomment les amandes ou pâte de péké contre 95,7\% ( $p<0,001)$ qui consomment les noix de palme ou la mwambe, dans une proportion comparable $(96,4 \% ; p<0,001)$, à ceux qui affectionnent des graines de la pâte d'arachide $89,2 \%$; $p<0,001$ consomment le safou, $87,8 \%$ consomment les graines ou pâte de courge et seulement $9,2 \% ; p<0,001$ goûtent à autre chose.

\section{A. Cas du Gabon}

Tableau 3 : Pratiques des ménages et consommation des oléagineux

\begin{tabular}{l|l|l|l|l}
\hline & Caractéristiques & Effectif & $\%$ & $\begin{array}{l}\text { Valeur de } \mathbf{x} \\
\text { et de } p\end{array}$ \\
\hline \multirow{2}{*}{ Ménages transformant ou non les oléagineux } & Oui & 141 & 64,7 & $x 2=41,706$ et \\
& Non & 77 & 35,3 & $p=0,00000$ \\
& Total & 218 & 100 & \\
\hline \multirow{3}{*}{ Types d'oléagineux transformés } & Noix de palme & 141 & 64,7 & $x 2=41706$ et \\
& Non spécifié & 77 & 35,3 & $p=0,00000$ \\
& Total & 218 & 100 & \\
\hline \multirow{2}{*}{$\begin{array}{l}\text { Connaissance des transformateurs } \\
\text { environnants }\end{array}$} & Oui & 59 & 27,1 & $x 2=26,860$ et \\
& Non & 148 & 67,9 & $p=0,00000$ \\
& Non spécifié & 11 & 5 & \\
\hline
\end{tabular}




\section{Boundena et al. J. Appl. Biosci. 2016 Enquête de consommation alimentaire en zone CEMAC : Cas de la}

république du Congo et du Gabon

Le Tableau 3 montre au total que $64,7 \%(p<0,000)$ des sujets enquêtés transforment les oléagineux Au Gabon aussi, une grande majorité des sujets enquêtés $(67,9 \%$; $p<0,000)$ ne se soucient pas d'avoir des connaissances sur les produits consommés

Tableau 4 : Principaux types d'oléagineux consommés à l'état brut ou semi brut

\begin{tabular}{|c|c|c|c|c|}
\hline & Caractéristiques & Effectif & $\%$ & Valeur de $x 2$ et de $p$ \\
\hline Amandes et/ou pate d'odika & \begin{tabular}{|l|} 
Oui \\
Non \\
Total
\end{tabular} & $\begin{array}{l}204 \\
14 \\
218\end{array}$ & $\begin{array}{l}93,6 \\
6,4 \\
100\end{array}$ & $\begin{array}{l}x^{2}=63950 \text { et } \\
p=0,00000\end{array}$ \\
\hline Noix de palme et/ou nyembwe & $\begin{array}{l}\text { Oui } \\
\text { Non } \\
\text { Non spécifié } \\
\text { Total }\end{array}$ & $\begin{array}{l}205 \\
12 \\
1 \\
218\end{array}$ & $\begin{array}{l}94 \\
5,5 \\
0,5 \\
100\end{array}$ & $\begin{array}{l}x^{2}=59565 \text { et } \\
p=0,00000\end{array}$ \\
\hline Graines et/ou pate d'arachide & $\begin{array}{l}\text { Oui } \\
\text { Non } \\
\text { Non spécifié } \\
\text { Total }\end{array}$ & $\begin{array}{l}193 \\
19 \\
6 \\
218\end{array}$ & $\begin{array}{l}88,5 \\
8,7 \\
2,8 \\
100\end{array}$ & $\begin{array}{l}x^{2}=39990 \text { et } \\
p=0,00000\end{array}$ \\
\hline Graines et/ou pate de courge & $\begin{array}{l}\text { Oui } \\
\text { Non } \\
\text { Non spécifié } \\
\text { Total }\end{array}$ & $\begin{array}{l}172 \\
39 \\
7 \\
218\end{array}$ & $\begin{array}{l}78,9 \\
17,9 \\
3,2 \\
100\end{array}$ & $\begin{array}{l}x^{2}=36755 \text { et } \\
p=0,00000\end{array}$ \\
\hline Atanga & $\begin{array}{l}\text { Oui } \\
\text { Non } \\
\text { Non spécifié } \\
\text { Total }\end{array}$ & $\begin{array}{l}209 \\
4 \\
5 \\
218\end{array}$ & $\begin{array}{l}95,9 \\
1,8 \\
2,3 \\
100\end{array}$ & $\begin{array}{l}x 2=48159 \mathrm{et} \\
p=0,00000\end{array}$ \\
\hline Autres oléagineux & $\begin{array}{l}\text { Oui } \\
\text { Non } \\
\text { Non spécifié } \\
\text { Total }\end{array}$ & $\begin{array}{l}124 \\
46 \\
48 \\
218\end{array}$ & \begin{tabular}{|l|}
56,9 \\
21,1 \\
22 \\
100 \\
\end{tabular} & $\begin{array}{l}x 2=29776 \text { et } \\
p=0,00000\end{array}$ \\
\hline
\end{tabular}

D'après le tableau 4 , on constate que $93,6 \%$ des ménages gabonais consomment les amandes d'odika $(n=204 ; p=0,00000)$ De même, 94\% d'entre eux consomment les noix de palme et/ou nyembwè Dans une proportion comparable $88,5 \% ; p=0,00000$, il s'agit des

Modalités d'approvisionnement et de consommation des oléagineux locaux à l'état brut ou semi brut par les ménages enquêtes : Cas de la noix de palme Le tableau 5 montre que dans les ménages, la dernière consommation des noix de palme ou de la mwambe datant moins d'une semaine est de $35,3 \%$ et celui datant graines et/ou pâte d'arachide II est de même pour l'Atanga avec 95,9\%; $p=0,00000$ Dans le même sens, $78,9 \%$ des ménages consomment les graines ou les pâtes de courges

la semaine est de $17,3 \%$ des cas $(p<0,02)$. Le mode d'achat d'acquisition des noix est majoritairement l'achat $(89,9 \% ; p<0,001)$, et le lieu d'acquisition le grand marché $(89,9 \% ; p<0,001)$. Les détaillants du marché en est davantage $(86,3 \% ; p<0,001)$ les fournisseurs. 
Boundena et al. J. Appl. Biosci. 2016 Enquête de consommation alimentaire en zone CEMAC : Cas de la république du Congo et du Gabon

\section{A. CAS DU CONGO}

Tableau 5 : Modalités d'approvisionnement en noix de palme et mwambe

\begin{tabular}{|c|c|c|c|c|}
\hline & Caractéristiques & Effectif (n) & $\%$ & Valeur de $x^{2}$ et $p$ \\
\hline $\begin{array}{l}\text { Dernière consommation de la noix de } \\
\text { palme ou la mwambe par les } \\
\text { ménages enquêtés }\end{array}$ & $\begin{array}{c}\text { Moins d'une semaine } \\
\text { Une semaine } \\
\text { Deux semaines } \\
\text { Plus de deux semaines } \\
\text { Nsp }\end{array}$ & $\begin{array}{l}49 \\
24 \\
18 \\
41 \\
7\end{array}$ & $\begin{array}{l}35,3 \\
17,3 \\
12,9 \\
29,5 \\
5,0\end{array}$ & $\begin{array}{l}\text { So }=19,884 \\
P<0,02\end{array}$ \\
\hline $\begin{array}{l}\text { Modalités d'approvisionnement en } \\
\text { noix de palme des ménages } \\
\text { enquêtés }\end{array}$ & $\begin{array}{c}\text { Achat } \\
\text { Verger familial } \\
\text { Don d'un parent } \\
\text { Autres }\end{array}$ & $\begin{array}{l}125 \\
6 \\
1 \\
7\end{array}$ & $\begin{array}{l}89,9 \\
4,3 \\
0,7 \\
5,0\end{array}$ & $\begin{array}{l}\text { So }=29,973 \\
P<0,001\end{array}$ \\
\hline $\begin{array}{l}\text { Lieu d'approvisionnement en noix de } \\
\text { palme }\end{array}$ & $\begin{array}{l}\text { Grand marché } \\
\text { Marché de rue } \\
\text { Autres }\end{array}$ & $\begin{array}{l}125 \\
2 \\
12\end{array}$ & $\begin{array}{l}89,9 \\
1,4 \\
8,6\end{array}$ & $\begin{array}{l}\text { So }= \\
35,271 \\
P<0,001\end{array}$ \\
\hline Fournisseur de noix de palme & $\begin{array}{l}\text { Auprès d'un récolteur } \\
\text { Détaillant au marché } \\
\text { Autres }\end{array}$ & $\begin{array}{l}7 \\
120 \\
12\end{array}$ & $\begin{array}{l}5,0 \\
86,3 \\
8,6\end{array}$ & $\begin{array}{l}\text { So }=36,953 \\
P<0,001\end{array}$ \\
\hline $\begin{array}{l}\text { Fréquentation des lieux } \\
\text { d'approvisionnement }\end{array}$ & $\begin{array}{l}\text { Même endroit } \\
\text { Changement d'endroit } \\
\text { Autres }\end{array}$ & $\begin{array}{l}14 \\
113 \\
12\end{array}$ & $\begin{array}{l}10,1 \\
81,3 \\
8,6\end{array}$ & $\begin{array}{l}\text { So }=33,287 \\
P<0,001\end{array}$ \\
\hline
\end{tabular}

Cependant, les lieux d'approvisionnement sont variables $(81,3 \% ; p<0,001)$. Par rapport à la flambée des prix, la figure 1 (ci-après) présente les modalités d'achat et quantités associées par les ménages et les motifs de choix et prix d'achat La figure $1 \mathrm{~A}$ indique que, les noix de palme sont souvent achetés par tas $(90,6 \% ; p<0,001)$. Quant à la quantité de noix par cuisson, pour un plat, elle varie entre deux et cinq tas $(66,9 \%$ des cas ; $p<0,001)$.
Celle-ci varie dans 8 cas sur 10 selon les besoins. La figure 1B montre que la couleur des noix de palme représente le facteur primordial de choix des noix de palme $(77,0 \% ; p<0,001)$. Par contre, le prix moyen d'achat par unité, selon le ménage varie entre $50 \mathrm{~F}$ et 200 FCFA soit $37,4 \%$ pour $50-100 \mathrm{~F}$ et $26,6 \%$ pour $150-200$ $F(p<0,02)$.

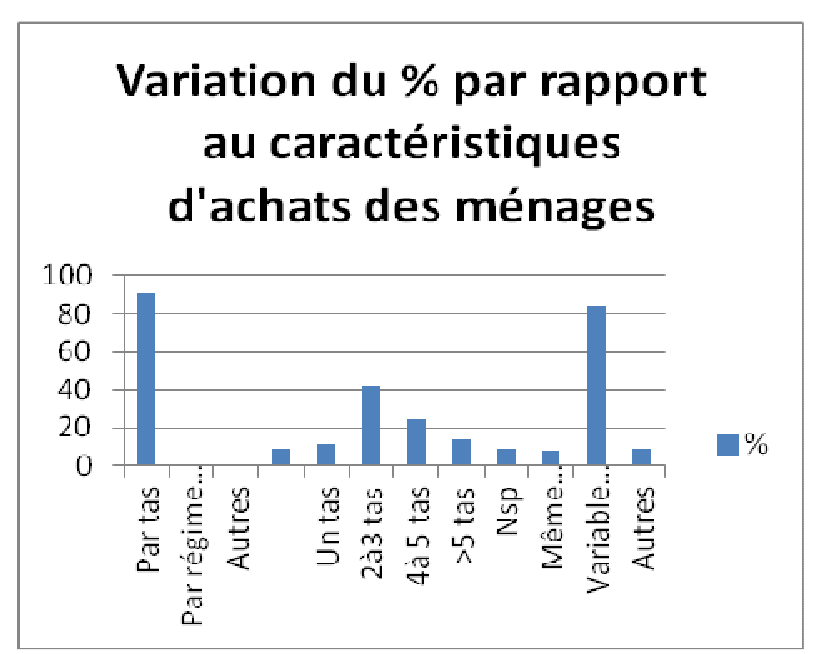

A : Modalités d'achat et quantité associée

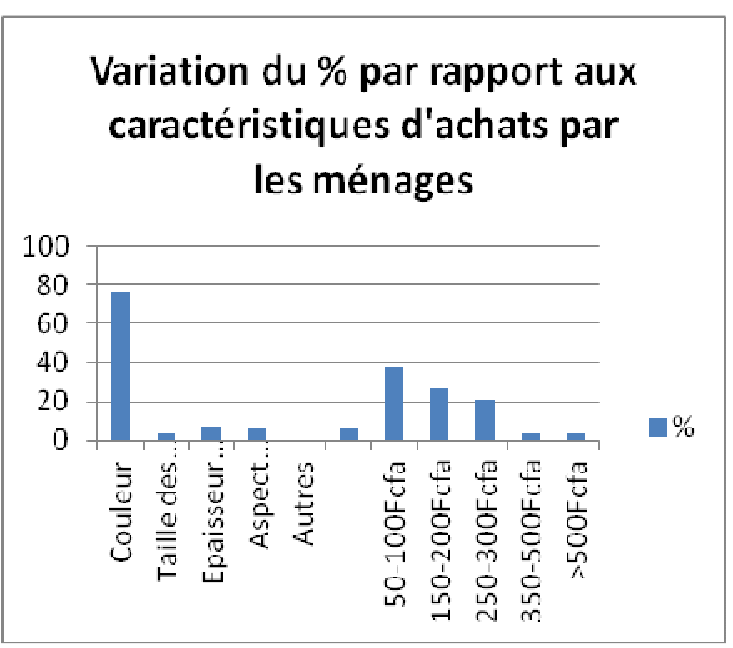

$B$ : Motifs de choix et prix d'achat

Figure1 : Variation du pourcentage par rapport aux modalités et motifs de choix au Congo 


\section{B. CAS DU GABON}

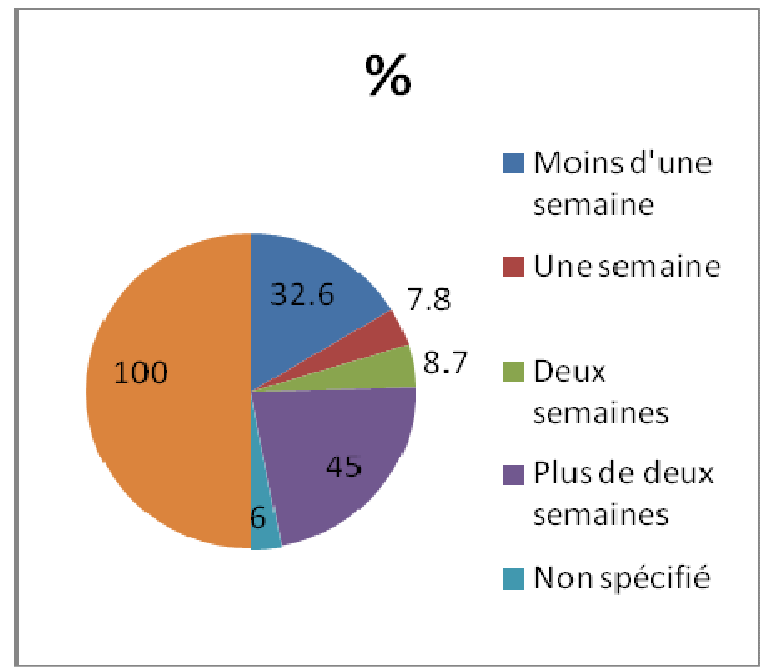

A : Dernière consommation des noix de palme et/ou nyembwè par les ménages enquêtés

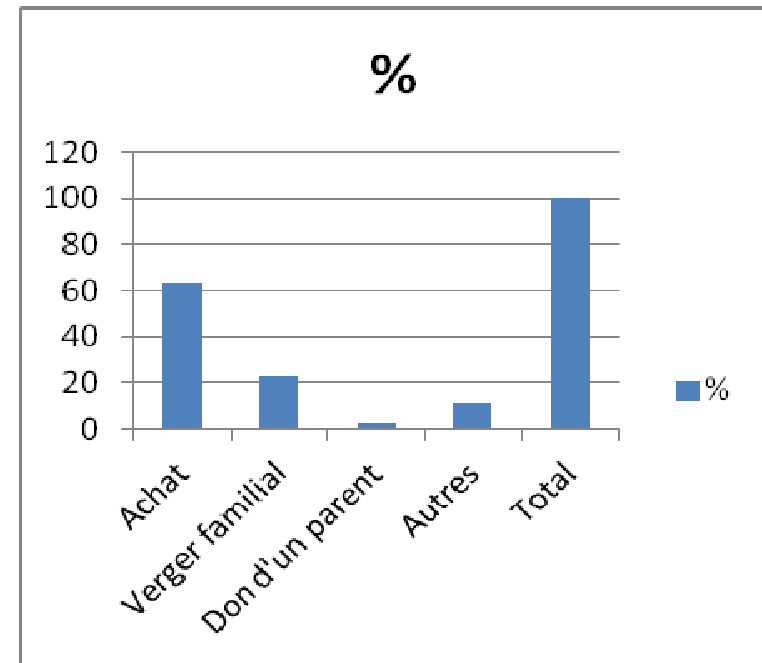

$B$ : Modalités d'approvisionnement en noix de palme des ménages enquêtés

Figure 2 : Modalités d'approvisionnement en noix de palme et/ou Nyembè (cas du Gabon)

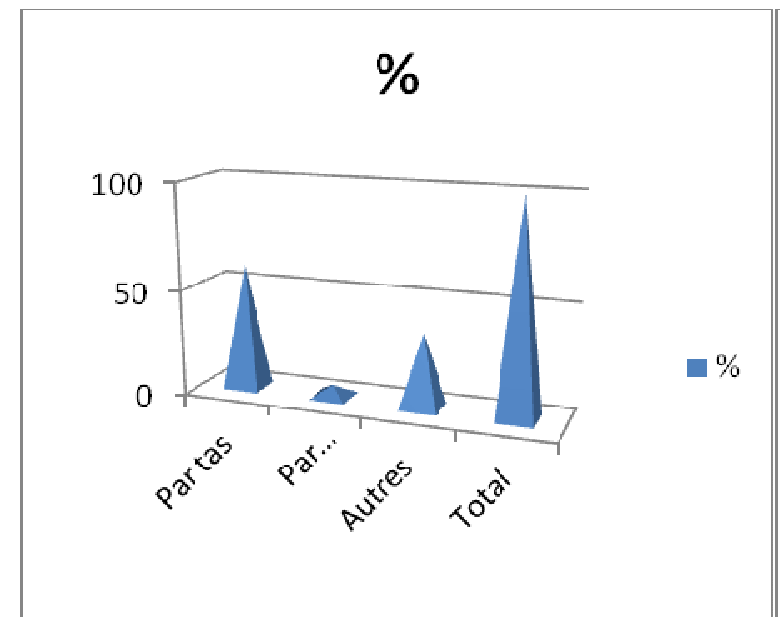

A : Modalités d'achat de noix de palme par les ménages enquêtés

Figure 3 : Modalités d'achat et quantité associée (cas du Gabon)

Sur 218 ménages enquêtés au Gabon, 64,7\% consomment les noix de palme contre $35,3 \%$ qui n'en consomment pas. Cela prouve bien que l'huile de palme est ancrée dans les habitudes alimentaires dans cette région. Au Gabon, et dans la plupart des principales villes, on consomme beaucoup l'huile de palme que l'on en prépare artisanalement. Mais deux villes ont retenu toute notre attention, celles de Franceville et de Lambaréné. Lambaréné, capitale provinciale du MoyenOgooué, il existe une unité de production industrielle d'huile de palme qui co-existe avec de petites unités de production artisanale de l'huile de palme brute. De même dans la ville de Franceville, la plupart des ménages enquêtés traitent eux-mêmes les noix de palme. 


\section{Boundena et al . J. Appl. Biosci. 2016 Enquête de consommation alimentaire en zone CEMAC : Cas de la république du Congo et du Gabon}

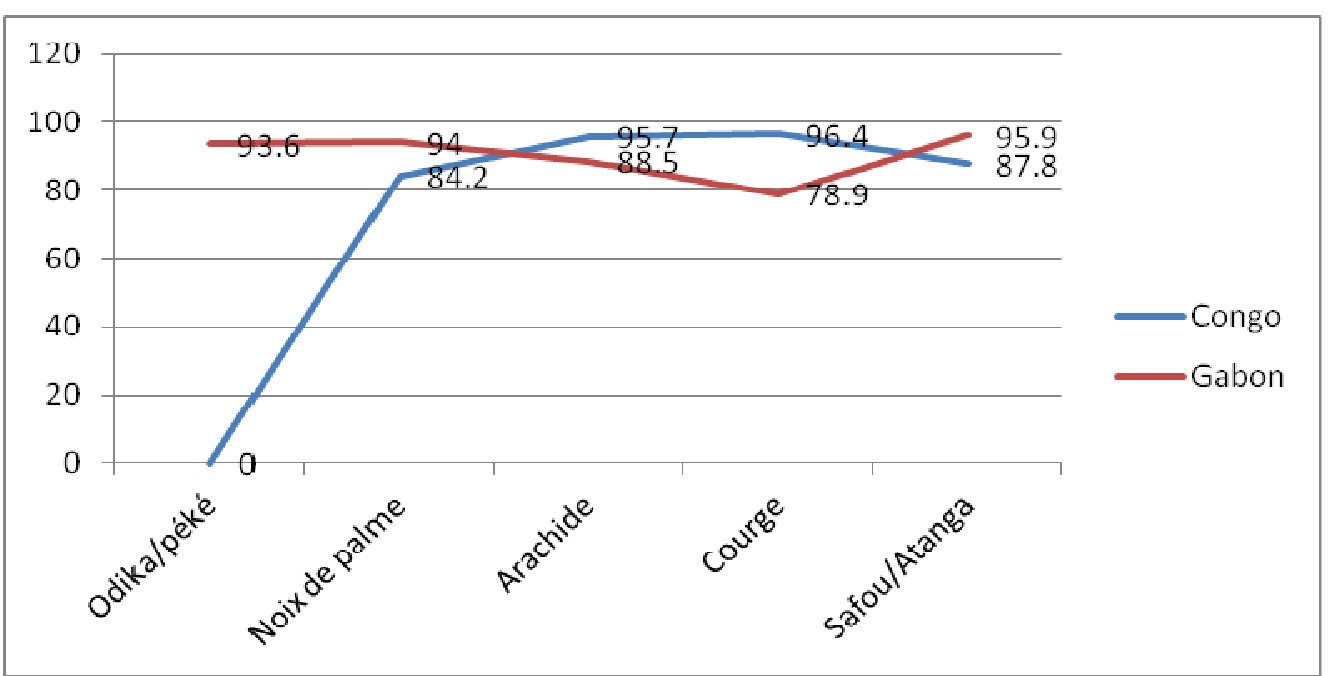

Figure 4 : Comparaison des principaux oléagineux consommés au Congo et au Gabon

\section{CONCLUSION}

Cette étude a tout d'abord montré que, d'un point de vue qualitatif, la comparaison des préférences alimentaires des deux enquêtes de consommation alimentaire au Congo et au Gabon et que pour un produit donné, il est appelé différemment selon le pays enquêté. C'est le cas par exemple du chocolat indigène qui est appelé péké au Congo et Odika au Gabon, tout comme la sauce de graine de l'huile de palme, qui est appelé mwambe au Congo et nyembwe au Gabon. II est apparu que les préférences d'apport en graisse ou oléagineux est très important dans ces deux pays. Mais les prix mondiaux des graines et des produits d'oléagineux exprimés en termes nominaux devraient se maintenir largement audessus des niveaux enregistrés dans les années à venir. La tendance récente à un resserrement progressif de l'offre et de la demande, et l'augmentation progressive des prix qui en résulte, commence à toucher les consommateurs des produits oléagineux, en particulier les ménages démunis des pays en développement Enfin dans les deux enquêtes les noix de palme (ou sauce de graine), le chocolat indigène, la pâte d'arachide sont très consommés dans les deux pays Les résultats de l'étude ont fournis les résultats suivant :

\section{REFERENCES BIBLIOGRAPHIQUES}

DEGBIA D, 2010 Étude de consommation urbaines sur les oléagineux et corps gras en zones CEMAC Cas de Brazzaville Mémoire de DEA P 19-24

FAO 2004a Évaluation des importations et aides alimentaires par rapport à l'appui au développement de l'agriculture : évidence
Pour le Congo, les ménages enquêtés consomment plus les noix de palme $(95,7 \%)$, la pâte d'arachide $(87,8 \%)$ et le safou $(89,2 \%)$;

Pour le Gabon, les ménages consomment plus les noix de palme (94\%), l'odika (93,6 \%), la pâte d'arachide $(88,5 \%)$ et la pâte de courge $(78,9 \%)$

Ces observations nous permettent d'affirmer qu'ils ont un impact sur le comportement des consommateurs, des producteurs et des autres acteurs du marché à l'intérieur des pays, ainsi que ceux des négociants internationaux, et, à terme, elles induisent des ajustements au niveaux de la production d'oléagineux. De plus cette enquête, au Congo et au Gabon, semblait être la période $(2010,2013$ et 2014) la plus critique en termes de diversité alimentaire et d'apports lipidiques. Néanmoins, si les mêmes tendances qualitatives se dégagent dans les deux enquêtes, des différences quantitatives notoires concernant les prévalences de consommateurs ont été mises en évidence. Dans la plupart des cas, ces disparités ne sont pas très significatives (en termes de différences ou d'écarts), mais il est apparu qu'elles pourraient être en partie expliquées par les origines ethniques de chacun des deux pays enquêtés.

par l'étude de cas, République du Tchad Organisation des Nations Unies pour l'alimentation et l'agriculture Rome (Disponible à http://wwwfaoorg/tc/Tca/ work05/Tchadpdf) 
Boundena et al. J. Appl. Biosci. 2016 Enquête de consommation alimentaire en zone CEMAC : Cas de la république du Congo et du Gabon

FAO, 2009 Profil nutritionnel du Tchad Division de la nutrition et de la protection des consommateurs PP 3

FAO, 2011 Perspectives agricoles de l'OCDE et de la FAO 2011-2020
MONTFORT M-A, 2005 Filières oléagineuses africaines Notes et études économiques PP $55-85$

RGPH, 2010 Recensement Générale de la Population Humaine Congo 